\title{
Effets collectifs des moteurs moléculaires : fonctions biologiques des oscillations mécaniques
}

Daniel Riveline ${ }^{(1,2)}$ (riveline@unistra.fr) et Karsten Kruse $\mathbf{e}^{(3)}$

(1) Laboratoire de Physique Cellulaire, ISIS/IGBMC, Université de Strasbourg et CNRS (UMR 7006),

8 allée Gaspard Monge, 67083 Strasbourg

(2) Biologie du développement et cellules souches, IGBMC, CNRS (UMR 7104), INSERM (U964), Université de Strasbourg,

1 rue Laurent Fries, BP 10142, 67400 IIIkirch

(3) Theoretische Physik, Universität des Saarlandes, Postfach 151150, 66041 Saarbrücken, Allemagne

L'étude physique de la

matière vivante montre

l'importance en biologie

de la génération spontanée

de contraintes. Longtemps

négligés, ces phénomènes

ont suscité un regain d'intérêt

auprès des biologistes

du développement depuis

une dizaine d'années.

Les forces sont générées par

des effets collectifs de moteurs

nanométriques, et dictent

les changements de formes

des cellules, des tissus

et des embryons. En particulier,

des oscillations mécaniques

spontanées peuvent se

développer dans la matière

vivante à toutes échelles.

Dans cet article, nous en

illustrons les conséquences

pour la perception de forces,

et dans la morphogénèse

chez les embryons.

Les termes en italiques suivis par un astérisque

sont définis dans le glossaire, p. 9.
Dans un texte visionnaire, Aristote cherche à définir ce qui caractérise et distingue la nature - matière vivante - de la matière inerte. Il pressent l'existence d'un principe du mouvement :

«De tout ce que nous venons de dire, il résulte que la nature, dans son sens primitif et fondamental, c'est l'essence des êtres qui ont, en eux-mêmes et en tant que tels, le principe de leur mouvement. La matière, en effet, ne prend le nom de nature que parce qu'elle est susceptible de recevoir en elle ce principe ; et le devenir et la croissance, que parce que ce sont des mouvements procédant de ce principe. La nature, en ce sens, est le principe du mouvement des êtres naturels, immanent en quelque sorte, soit en puissance, soit en acte. " (Aristote, Métaphysique, Livre V).

Richard Feynman envisage, dans les années 1950, l'existence de moteurs à l'échelle de la molécule, qui pourraient avoir des mouvements directionnels et extraire $\mathrm{du}$ travail dans un environnement soumis au bruit thermique, en analogie avec une roue à rochet ${ }^{\star}$. Les moteurs moléculaires ${ }^{\star}$ ont, par la suite, été découverts par A.F. Huxley et H.E. Huxley en 1954. En biologie, ils sont omniprésents à toutes les échelles : dans la traduction de l'information codée dans le génome, dans le transport de vésicules dans les cellules, dans l'application de forces dans les muscles, ou encore dans le changement de formes des tissus et des organes pendant le développement des embryons. L'idée d'Aristote sur cette matière qui prend le nom de nature était juste : les moteurs moléculaires contrôlent le devenir et la croissance de la matière vivante.
Ainsi, les contractions musculaires sont provoquées par des myosines, moteurs moléculaires, qui sont en contact avec des polymères partenaires - les filaments d'actine [1]. Ce travail mécanique est possible grâce à l'énergie chimique libérée lorsque la myosine hydrolyse l'Adénosine TriPhosphate (ATP) (cette énergie chimique provient de la lumière solaire chez les végétaux et de l'alimentation chez les animaux). Ce même mécanisme moléculaire à la base de la contraction musculaire provoque aussi les changements de forme des cellules, des tissus et des organes en développement.

La matière, ainsi munie du pouvoir de transformer sur une échelle locale l'énergie chimique en énergie mécanique, présente des propriétés extraordinaires, inconnues pour la matière inerte communément étudiée en physique. Ces matériaux polaires actifs peuvent générer des flux spontanés, des contractions et des oscillations spontanées. L'adjectif " polaire » fait référence à la polarité des filaments d'actine, qui provient des différences structurales de ses deux extrémités appelées arbitrairement + et - ; cette caractéristique peut conduire à une polarité macroscopique du gel. "Actif» fait référence à la présence de moteurs utilisant l'énergie chimique. Le cytosquelette ${ }^{\star}$ de chaque cellule comprend l'enchevêtrement de plusieurs paires de ces protéines - polymères polaires/moteurs : cette structure dynamique dicte les changements de formes des cellules et des tissus in vivo.

Dans cet article, nous illustrons les propriétés de la matière active en présentant les oscillations observées pour une variété de systèmes, allant du niveau moléculaire 


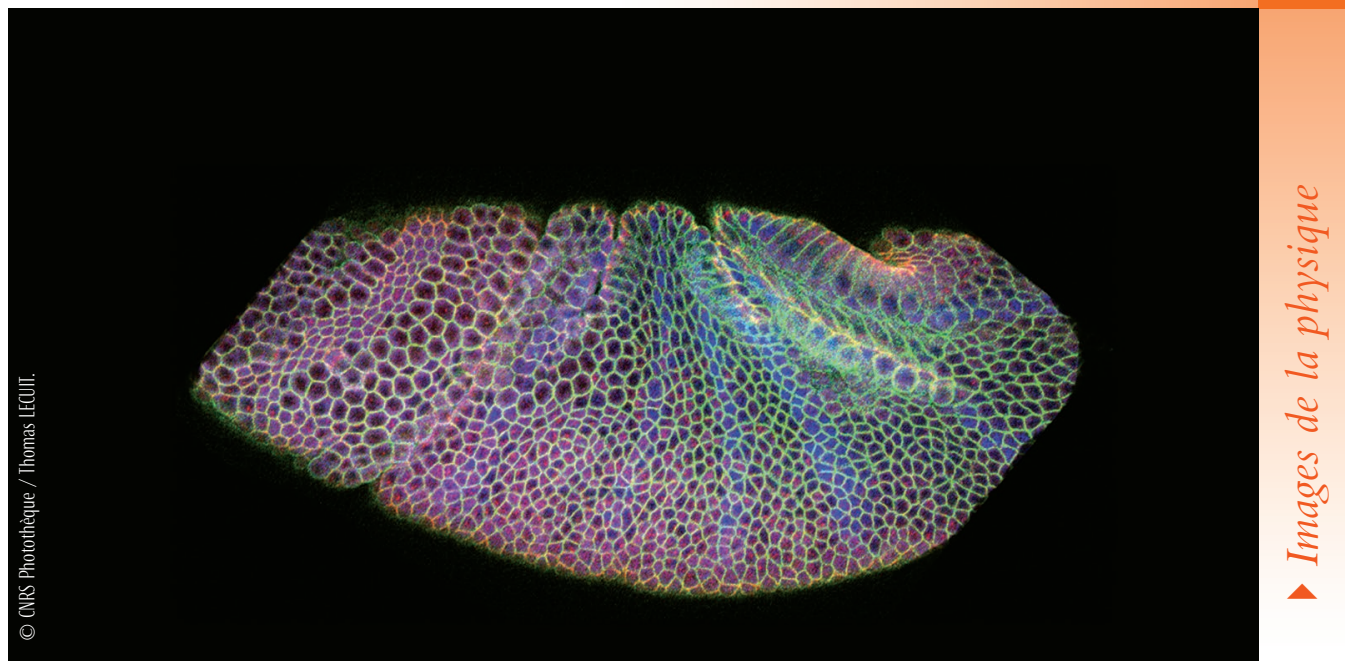

Embryon de drosophile : en bleu, contour des cellules épithéliales. Un des acteurs indispensables au réarrangement des cellules au cours de l'embryogenèse est la myosine II, un moteur moléculaire. Image obtenue par marquage fluorescent des jonctions entre cellules, au Laboratoire de génétique et physiologie du développement (UMR CNRS 6545).

pour quelques moteurs jusqu'au tissu. Bien que les longueurs et temps caractéristiques different, ces oscillations ont toutes comme origine les effets collectifs de moteurs qui peuvent spontanément générer des mouvements périodiques. Nous donnons d'abord des éléments sur les moteurs moléculaires et sur le déclenchement des oscillations mécaniques spontanées lorsque plusieurs moteurs sont couplés mécaniquement. Nous décrivons dans une seconde partie les phénomènes d'oscillation dans les cellules individuelles pour la mécanosension ${ }^{\star}$, et dans les cellules composant les tissus pour les embryons en développement, où plusieurs phénomènes se produisent : le nombre de cellules augmente et des réorganisations permanentes des tissus permettent des changements de leurs formes. Ces morphogénèses ne sont pas seulement attribuables à la génétique, mais aussi à la génération de contraintes dues au cytosquelette et aux moteurs associés.

\section{I - Les moteurs moléculaires et les oscillations}

\section{Les moteurs moléculaires sont bien compris.}

Schématiquement, un moteur moléculaire tel que la myosine peut être représenté comme une double tête prolongée par une queue (fig. 1). C'est avec la tête que le moteur s'attache au filament. Le principe du mouvement d'un moteur moléculaire repose sur le changement de conformation de la tête, qui est associé à l'hydrolyse de l'ATP (fig. 1a). Ces changements ont été bien caractérisés d'un point de vue biochimique.
Par exemple, pour la myosine musculaire, la fixation d'une molécule d'ATP libère la tête du moteur du filament d'actine auquel il était attaché. Simultanément, la tête " pivote ". Après l'hydrolyse de l'ATP, la tête s'attache à nouveau au filament. La libération $\mathrm{du}$ groupe phosphate $\mathrm{P}_{\mathrm{i}}$ induit un autre changement de conformation qui génère une contrainte sur le moteur moléculaire, engendrant un mouvement du moteur vers une extrémité déterminée du filament, toujours la même.

Notre compréhension des moteurs moléculaires a profité considérablement des tests in vitro. Ces tests de motilité sont conçus pour observer le mouvement directionnel des moteurs sur des surfaces par microscopie optique. Par exemple, les myosines sont greffées sur une surface de verre et des filaments d'actine marqués par fluorescence sont observés en présence d'ATP : ils avancent de manière directionnelle à la manière de vers de terre, et toujours dans le même sens ; l'avant des filaments "cherche " des myosines avec lesquelles interagir, puis s'engage dans une direction (fig. 1b). Les myosines associées au filament hydrolysent l'ATP, changent de conformation, et peuvent ainsi appliquer des forces sur le filament. Le montage " inverse » est équivalent : des myosines peuvent se déplacer sur des filaments d'actine greffés sur des surfaces.

Pour la description des propriétés physiques des moteurs moléculaires individuels, de nombreux outils ont été utilisés. En particulier, les pinces optiques ont permis de mesurer la relation force-vitesse de moteurs individuels. Ces études ont bien caractérisé la physique des moteurs moléculaires
$-$

(1)

(2)
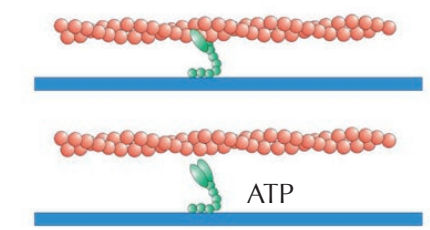

(3)

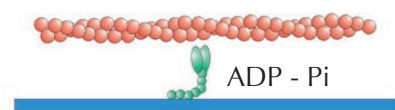

(4)

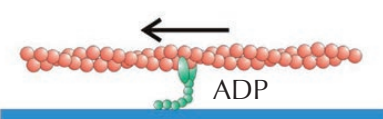

(5)
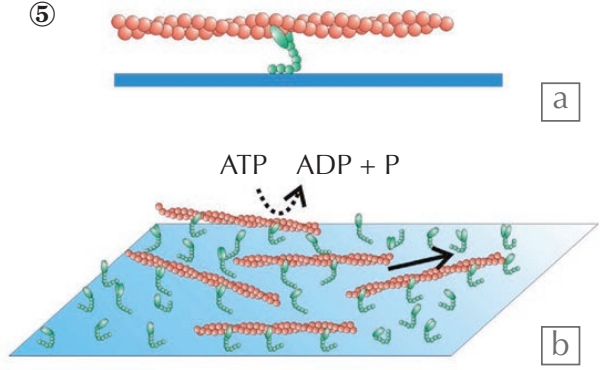

\section{Moteurs moléculaires}

(a) Les filaments d'actine (en rouge) sont des polymères de $7 \mathrm{~nm}$ de diamètre ; leur symétrie est brisée et les extrémités sont notées + et - de manière conventionnelle. Les myosines (en vert) sont des moteurs moléculaires. La myosine passe par des cycles d'hydrolyse d'ATP (ATP $+\mathrm{H}_{2} \mathrm{O} \rightarrow \mathrm{ADP}+\mathrm{P}_{\mathrm{i}}+$ énergie), durant lesquels elle se fixe (1) et se détache des filaments $(2,3)$, tout en changeant de conformation et se reliant au filament sur un monomère plus proche de l'extrémité + $(3,4)$. La myosine fixée au verre (en bleu) donne un « coup » et le filament se déplace vers la gauche $(4,5)$.

(b) Sur une surface recouverte de têtes de myosine, les filaments d'actine se déplacent de manière directionnelle en présence d’ATP. Les flèches continues sur les figures a et b indiquent les déplacements résultants. 
$>>$

uniques en interaction avec des filaments. Ainsi, la force appliquée par le moteur est de l'ordre du piconewton, le pas de déplacement est de l'ordre du nanomètre.

Cette image moléculaire est bien utile pour décrire l'origine du mouvement, mais elle masque les principes physiques impliqués dans le déplacement. Étant baigné dans l'eau, le moteur est soumis au mouvement brownien. Comment une molécule peut-elle avancer d'une manière directionnelle dans de telles conditions ? Deux caractéristiques essentielles sont requises : la polarité des monomères constituant les filaments, qui peut être représentée par un potentiel d'interaction asymétrique et périodique ${ }^{\star}$ avec les moteurs, ainsi qu'une source d'énergie qui met le système hors d'équilibre. Pour le comprendre, un modèle simple a été proposé. Il s'agit d'une particule qui est exposée à un potentiel qui change entre deux états $W_{1}$ et $W_{2}$ (fig. $2 a$ ) : la myosine est attachée au filament d'actine dans l'état $\mathrm{W}_{1}$, et détachée dans l'état $\mathrm{W}_{2}$ (d'autres exemples sont envisageables). Les transitions entre les deux états du potentiel correspondent aux séquences d'attachement et de détachement du moteur liées au cycle d'hydrolyse de l'ATP. En bref, on a un potentiel périodique et asymétrique d'interaction de la myosine et du filament d'actine : l'hydrolyse de l'ATP fait sortir la myosine des puits de potentiel $\mathrm{W}_{1}$, elle diffuse dans $\mathrm{W}_{2}$; la myosine est ensuite soumise à $W_{1}$ puis " retombe » préférentiellement d'un côté du potentiel d'interaction, à cause de son asymétrie : elle a statistiquement un mouvement global moyen.

\section{Un ensemble de moteurs}

se comporte différemment

de la somme de moteurs individuels.

On l'a rappelé, un moteur moléculaire donné fait toujours avancer le filament dans la même direction. Mais quand plusieurs moteurs agissent ensemble sur le même filament, le mouvement collectif peut être très différent de la simple somme des actions individuelles des moteurs. De manière remarquable, il a été montré expérimentalement qu'un ensemble de moteurs peut conduire à des déplacements bidirectionnels. Couplé à un ressort, ce phénomène peut engendrer des oscillations spontanées, ce que nous allons détailler.

Expérimentalement, Riveline et al. [3] ont démontré en 1998 un tel mouvement bidirectionnel, en utilisant un test de
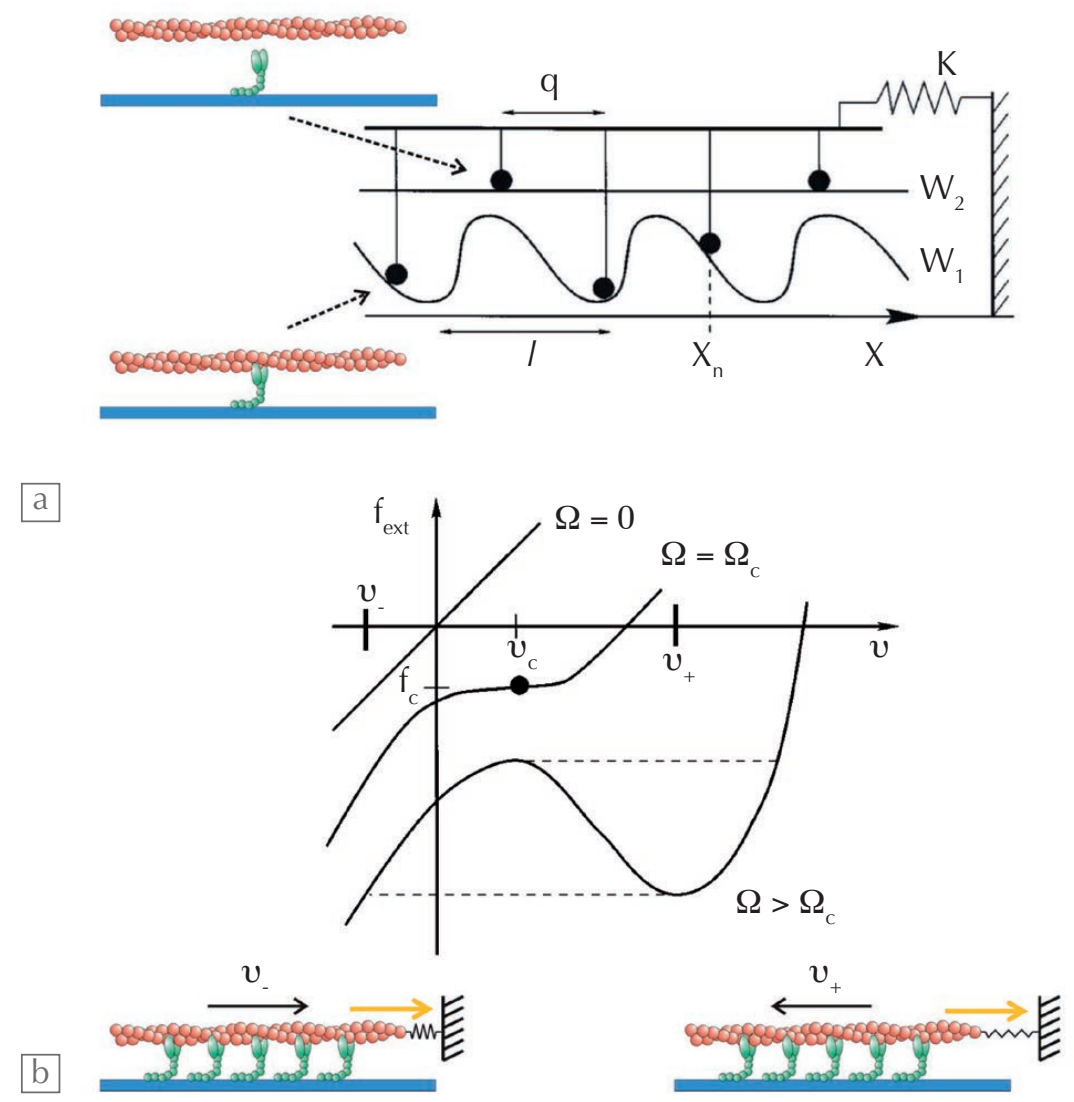

2. Description générique de la dynamique d'un ensemble de moteurs [2]

(a) Les têtes des moteurs sont représentées par des disques noirs distants d'une longueur q et connectés à une structure. Les moteurs peuvent être couplés de manière rigide. L'interaction d'une tête motrice avec le filament de période I à différents états peut être décrite par des potentiels $W_{1}$ et $W_{2}$. Les moteurs vont toujours aller vers le minimum du potentiel. Ils transitent entre les potentiels de manière stochastique. L'activité hors équilibre (voir texte) est traduite par $\Omega$. La structure est liée à un ressort. Des schémas représentent les états de fixation de myosines individuelles sur l'actine dans chacun des deux états. Noter que les périodes I et q sont différentes, et que les myosines ne sont donc pas nécessairement toutes aux minima du potentiel $W_{1}$.

(b) Représentation schématique d'une instabilité dynamique ; le diagramme force-vitesse résultant d'un modèle est donné. Pour une activité $\Omega$ supérieure à une valeur critique $\Omega_{0}$, l'ensemble peut se déplacer à deux vitesses de sens opposé avec la même force extérieure $\mathrm{f}_{\text {ext. }}$. Des schémas représentent les états de fixation des myosines sur un filament d'actine, avec une force de rappel représentée en orange et matérialisée par un ressort. Les flèches noires indiquent le sens de déplacement.
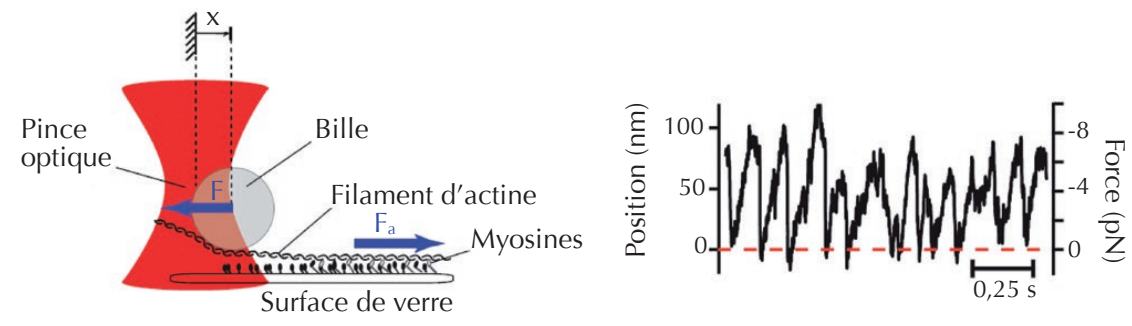

a

$\mathrm{b}$

\section{Dynamique d'un ensemble de moteurs in vitro}

(a) Des filaments d'actine individuels se déplacent sur un tapis de myosine et sont soumis à une force extérieure par une pince optique ; les myosines appliquent une force $\mathbf{F}_{\mathbf{a}}$, et la force de rappel $\mathbf{F}$ exercée par la pince est mesurée par la distance $x$ au centre du piège.

(b) La position $\mathrm{x}$ et la force de rappel $\mathbf{F}$ sont représentées en ordonnée : les filaments d'actine oscillent. (Voir Plaçais et al. [4] et les références associées à cette instabilité dans Kruse et Riveline [5]). 
motilité adapté ; une force extérieure s'opposait au déplacement des filaments tirés par les têtes de myosine ; deux vitesses étaient mesurées pour une même force extérieure. En 2009, un autre effet collectif sur le même système a été rapporté, en l'occurrence une oscillation : Plaçais et al. [4] ont utilisé une surface couverte de manière dense de myosines sur laquelle ils ont placé des filaments attachés à une bille de latex (fig. 3a). Cette bille était retenue par une pince optique. Ces pinces appliquaient une force de rappel, qui augmentait linéairement lorsque le filament d'actine se déplaçait. Le filament se mettait à osciller avec une fréquence d'une dizaine de hertz et une amplitude de $100 \mathrm{~nm}$ (fig. 3b). Dans ce dernier montage, les moteurs sont couplés via les filaments d'actine, mais la force est appliquée par l'intermédiaire d'un ressort (la pince optique) ; elle varie donc quand les moteurs déplacent le filament.

Pour comprendre ce phénomène d'oscillation, les moteurs sont représentés par des particules connectées entre elles, et pouvant transiter entre deux états d'énergies $W_{1}$ et $\mathrm{W}_{2}$ (fig. 2a); $\mathrm{W}_{1}$ représente un potentiel périodique` de période 1 , et $\mathrm{W}_{2}$ un potentiel plat dans lequel les particules peuvent diffuser ; l'activité $\Omega$ est reliée aux fréquences de transitions entre les deux états $\mathrm{W}_{1}$ et $\mathrm{W}_{2}$, et mesure la distance du système par rapport à l'état d'équilibre thermodynamique. Par exemple, un $\Omega$ élevé correspondra qualitativement à des excitations et des desexcitations plus fréquentes que ce que l'équilibre prédirait. Pour représenter la force mécanique extérieure, un ressort de raideur $\mathrm{K}$ s'oppose au déplacement de l'ensemble des particules. Lorsque l'activité de ces dernières dépasse un seuil $\Omega_{c}$, le diagramme force-vitesse montre des zones pour lesquelles deux vitesses coexistent pour une même force extérieure (fig. 2b).

Un mécanisme possible de ces oscillations est fondé sur la possibilité, décrite ci-dessus, de la coexistence de deux vitesses si les moteurs sont liés à un filament commun. Quel est l'effet d'un ressort sur l'ensemble de moteurs en interaction, pour des paramètres qui génèrent une coexistence de deux vitesses en sens opposés? Admettons que le filament avance sur l'une des deux branches du diagramme de la figure $2 b$. Le ressort se comprime par exemple. À partir d'un certain moment, le filament ne peut plus avancer et le ressort le force sur l'autre branche. Il se déplace alors dans l'autre sens jusqu'à ce que le ressort soit de nouveau chargé. Le filament s'arrête et change encore de branche. Cette instabilité dynamique est prédite par le modèle à deux états.

Un deuxième mécanisme possible est fondé sur le fait que le taux de détachement d'un moteur du filament dépend de la force appliquée. Le ressort comprimé induit une force sur tous les moteurs. Si l'un des moteurs se détache, les autres doivent se répartir la force qu'il supportait, ce qui augmente leur taux de détachement. Ainsi, une avalanche de détachements peut être déclenchée et le ressort se relâche. Ensuite, les moteurs s'attachent à nouveau au filament et le cycle recommence.

\section{\|l - Les oscillations associées aux systèmes acto-myosine dans les cellules et dans les embryons}

Ces oscillations mécaniques ont été observées in vitro pour une variété de systèmes expérimentaux, outre les complexes acto-myosine : par exemple, pour d'autres moteurs associés au squelette comme les kinésines ou les dynéines interagissant avec des microtubules - autres filaments polaires du cytosquelette. Elles ont aussi été observées dans plusieurs systèmes in vivo, depuis les mouvements périodiques d'éléments du cytosquelette dans des cellules individuelles, jusqu'aux contractions pulsées dans des embryons en développement. Ces phénomènes ont en commun de résulter d'une collection de plusieurs moteurs moléculaires agissant contre une force élastique résistante. La généralité de ce phénomène d'auto-organisation in vitro/in vivo est cohérente avec la nature générique du mécanisme d'oscillation discuté plus haut.

\section{Oscillations cellulaires in vivo}

Le battement des ailes se déroule chez certains insectes à une fréquence qui est plus grande que la fréquence maximale à laquelle un neurone peut déclencher un potentiel d'action. Les muscles d'insecte sont ainsi un exemple d'une oscillation mécanique spontanée observée in vivo. Les muscles sont composés d'un réseau très organisé de systèmes acto-myosine. L'unité contractile élémentaire est un sarcomère ${ }^{\star}$ (fig. 4a), et il a été établi que ceux-ci peuvent osciller spontanément dans des conditions physiologiques.
Pour les cellules non musculaires de mammiferes, la myosine II et les filaments d'actine peuvent s'assembler en fibres de stress ${ }^{\star}$, qui partagent avec les sarcomères l'arrangement linéaire de myosine et d'actine, avec une organisation moins régulière (voir encadré, p. 8). De manière remarquable, les fibres de stress sont observées dans des situations variées : elles ne s'assemblent pas seulement dans des cellules individuelles sur des surfaces, mais aussi entre des cellules voisines en culture, et dans des embryons en développement. Ces sortes de minimuscles sont notamment requis pour que les cellules se déplacent. Par ailleurs, il a été démontré que les cellules sondent leur environnement avec ces entités : en appliquant une force, les cellules peuvent renforcer localement les contacts adhésifs, ou contacts focau $x^{\star}$. Plus le contact est étendu avec une densité constante de colle, plus la force locale appliquée par la cellule est grande (voir encadré, p. 8). Ce phénomène nouveau de mécanosension a été montré à la fois pour les contacts entre les cellules et la matrice extracellulaire, et pour les contacts entre cellules voisines.

Les forces appliquées par la cellule sur ces zones d'adhésion ont été mesurées. On a montré qu'elles oscillent avec une période de l'ordre de 20 secondes (voir encadré, p. 8), c'est-à-dire durant un temps plus long que les oscillations de sarcomères isolés. Ce phénomène est lié aux fibres de stress qui appliquent des tensions sur les adhésions focales : les cellules ont localement des cycles de traction et de détachement qui sont cohérents avec les oscillations observées.

\section{Les oscillations mécaniques pendant} le développement embryonnaire

Un autre exemple d'oscillations liées aux complexes acto-myosine a été observé chez la mouche drosophile pendant une étape importante du développement embryonnaire (fig. 4b) : la fermeture de l'un de ses tissus - la fermeture dorsale ; les côtés latéraux de l'embryon se rapprochent pour se souder et ne former qu'un seul tissu. Deux parties d'un tissu cellulaire sont séparées par un domaine composé de cellules contractiles. Ce mouvement pulsé du tissu entraîne le déplacement du tissu voisin. Les câbles d'actine stabilisent la nouvelle organisation, relaxant le stress exercé par les cellules contractiles. Puis, ces cellules augmentent leur tension à nouveau avant une nouvelle stabilisation. 


\section{Mécanosension et oscillations}

La mécanosension est une propriété originale de la cellule vivante. Des fibres de stress sont associées à des points d'ancrage protéiques fixés à leur environnement, les contacts focaux. Lorsqu'elles appliquent des forces de cisaillement sur ces points d'attache (voir les schémas E1a et E1c, où le sens de la force appliquée par les fibres de stress sur le contact focal est représenté par une flèche), ces contacts adhésifs s'allongent. Une telle déformation est attendue, mais une autre caractéristique surprenante a été démontrée par Riveline et al. en 2001 [6] : la densité de " colle » est constante. Ce renforcement suggère que plus la force locale est élevée, plus I'adhésion est grande. Ce phénomène est différent de ce qu'on attend pour un système passif : sous traction locale, le contact devrait se décoller.

Comme les fibres de stress sont les applicateurs de forces (voir l'ensemble actine-myosine, fig. E1c), on peut s'attendre à ce qu'elles aient un comportement oscillant. Ces oscillations ont été démontrées par Plotnikov et al. en 2012 [7]. En visualisant les contacts par fluorescence (fig. E1a, photos de droite) et en mesurant la force locale le long des contacts individuels, il a été montré que la force oscillait sur chaque contact focal. La position de la force maximale sur le contact (le point rouge sur les photos de la figure E1a) varie également au cours du temps (courbe rouge sur la figure E1b). Cette oscillation semble ainsi contribuer à sonder localement l'environnement mécanique de la cellule sur une taille caractéristique de l'ordre de quelques micromètres.

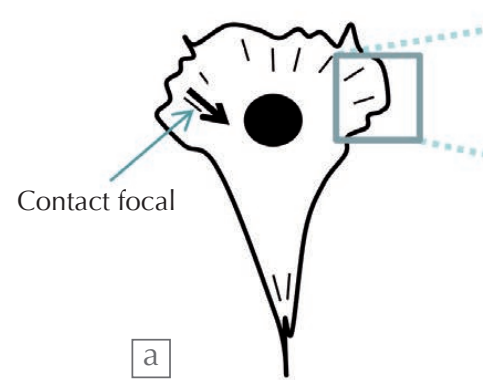

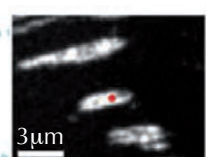

$0,00 \mathrm{~s}$

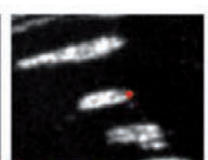

$0,05 \mathrm{~s}$

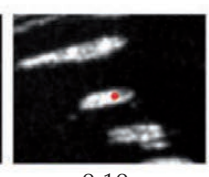

$0,10 \mathrm{~s}$
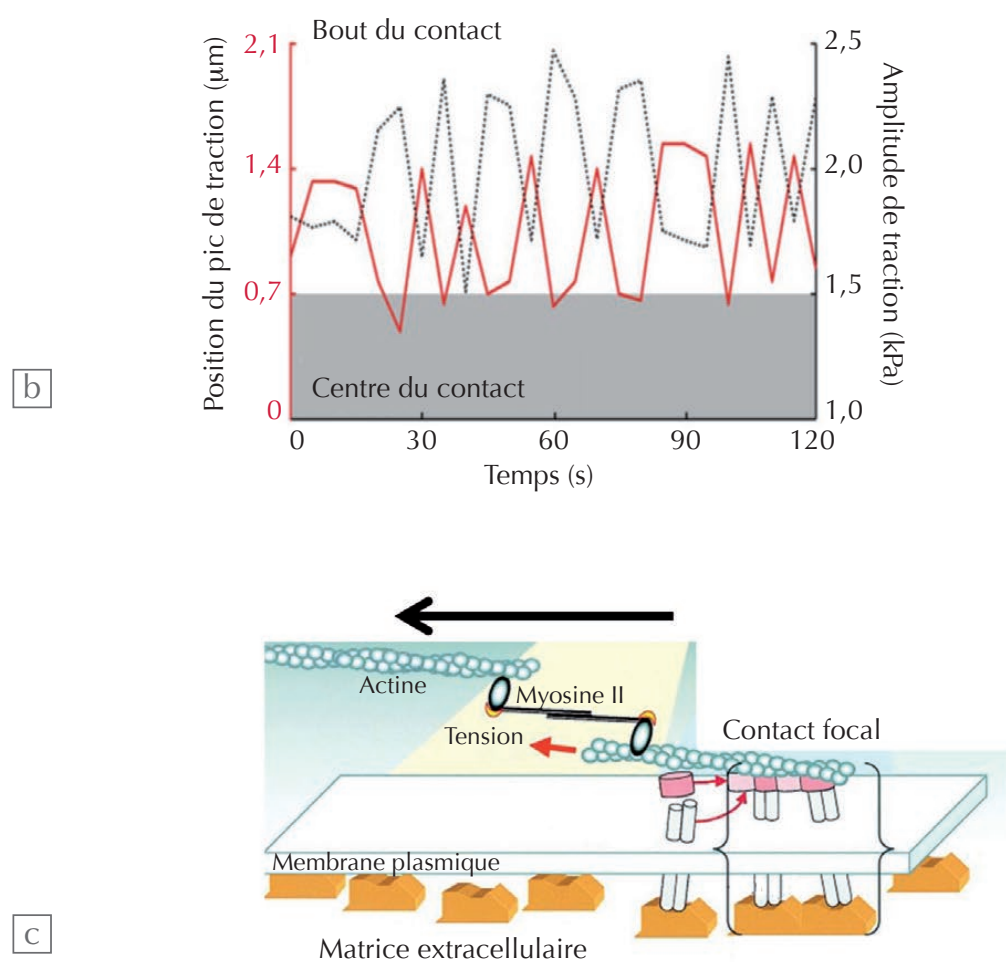

E1. (a, b) Comportement oscillant d'une fibre de stress [7].

(c) Application d'une contrainte sur la matrice extracellulaire par l'intermédiaire d'un contact focal. $\gg>$

La répétition de ce cycle mène à la fermeture totale du tissu. Ce rapprochement spatial menant à la fermeture d'un tissu biologique pourrait être modèle pour étudier le phénomène général de cicatrisation, qui montre des oscillations de l'ordre de la minute, dépendant des complexes acto-myosine.

Ces oscillations établissent des relations fortes entre les comportements de cellules individuelles et de celles des embryons en développement. Elles présentent des caractéristiques similaires : des amplitudes spatiales de l'ordre du micromètre (fig. 4c), des périodes temporelles de l'ordre de quelques minutes (fig. 4d) (à l'exception des oscillations musculaires qui sont bien plus rapides), et un rôle clé du cytosquelette d'acto-myosine. La similarité avec les expériences in vitro montrant les instabilités mécaniques est frappante. Cela suggère un mécanisme physique commun pour l'ensemble de ces phénomènes : la collection de moteurs myosine génère un stress dans chaque cellule et donc dans chaque tissu. Des composants élastiques intra- ou intercellulaires résistent à cette force résultante jusqu'à ce qu'un nouveau stress puisse être généré. Ainsi dans chaque situation, les cellules traversent des phases de contraction/ relaxation, de la même manière que des filaments individuels oscillent in vitro lorsqu'ils interagissent avec une collection de moteurs.

Il est clair que l'observation d'oscillations mécaniques cellulaires n'est pas suffisante pour conclure à un effet collectif de moteurs. Le diagramme de phase du système doit être mesuré pour qualifier la nature du phénomène oscillatoire. En particulier, les paramètres de contrôle mécanique doivent être variés, comme la densité des moteurs actifs, leurs activités, ou encore les propriétés élastiques du système. Dans des tissus, la force de rappel des cellules voisines pourrait être réduite en agissant sur les contacts adhésifs.

\section{Les fonctions des oscillations mécaniques pendant le développement}

Ces oscillations peuvent avoir une fonction biologique pendant le développement. D'abord, avec les fibres de stress, la cellule a la possibilité de sonder périodiquement la résistance de son environnement. Si la matrice ou les cellules voisines opposent 
une résistance, alors la cellule peut renforcer ses contacts adhésifs. Sinon, les contacts restent les mêmes ou disparaissent. Ainsi, le renforcement pourrait jouer un rôle important pour la détermination du destin d'une cellule. Par exemple, il a été montré que les formes et les divisions des cellules sont étroitement liées aux distributions des contacts adhésifs avec leur environnement, les contacts focaux. En oscillant, les fibres de stress permettraient aux cellules de sonder leur environnement mécanique avec une résolution temporelle de l'ordre de la minute. Par ailleurs, la phase de relaxation pourrait avoir un rôle spécifique, celui de relâcher la tension, donnant potentiellement la possibilité aux autres moteurs de se lier et aux fibres de stress d'appliquer des forces.

Le renforcement des adhésions entre cellules pendant les périodes des stress maximum pourrait figer la forme transitoire : les fibres de stress ne relaxeraient pas, grâce aux adhésions renforcées entre cellules. À la place, des forces plus grandes seraient appliquées dans le tissu modifié par ce nouveau stress. En retour, la nouvelle contraction pourrait promouvoir l'évolution des tissus pour continuer la modification. Ce mécanisme de blocage est bien représenté par la roue à rochet qui stabilise à chaque contraction.

\section{Conclusion}

Nous avons illustré des mécanismes d'oscillations documentés par des expériences in vitro et in vivo. Nous avons insisté sur les oscillations générées par le système actomyosine, car elles ont été démontrées par les tests de motilité, sur des cellules individuelles et sur des embryons en développement. Cette approche, nouvelle et exigeante, peut permettre d'expliquer des transitions en biologie du développement, au-delà des explications par la génétique et les réactions chimiques.

Nous remercions les collaborateurs passés et présents sur ces sujets de recherche, et en particulier Jacques Prost ; Gérard Laprie et Éva Riveline pour la citation d'Aristote, David Caballero et Jordi Comelles pour l'édition des figures. Cet article est une adaptation française de celui de Kruse et Riveline [5], où des références détaillées sont données.

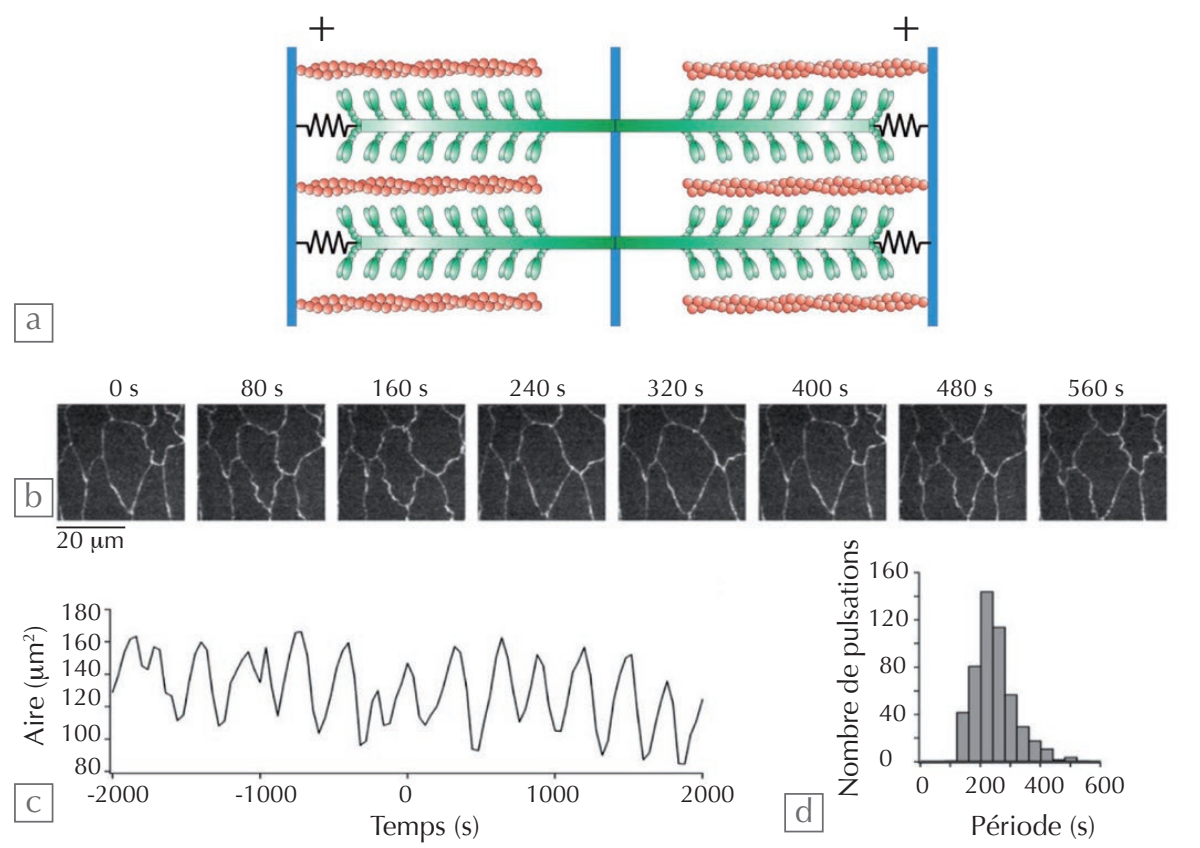

4. Les oscillations spontanées in vivo.

(a) Le sarcomère dans les muscles. Les filaments d'actine (en rose) sont ancrés à une surface (en bleu) par leurs extrémités +. Les moteurs myosines (en vert) interagissent avec les filaments ordonnés d’actine ; le muscle se contracte. (b) La fermeture du tissu dorsal chez la drosophile requiert des oscillations cellulaires : images successives du tissu dorsal, avec un marquage des frontières entre cellules ; les oscillations sont provoquées par les complexes acto-myosine et sont indispensables pour la morphogénèse [8].

(c) L'aire de la paroi cellulaire de la drosophile en fonction du temps montre des variations périodiques [8].

(d) Histogramme des périodes de pulsation [8].

\section{Glossaire}

Contacts focaux. Contacts adhésifs entre les cellules individuelles et leur environnement. Ces complexes protéiques ont une taille caractéristique de l'ordre du micromètre ; ils sont aussi liés au cytosquelette, et s'allongent sous contrainte mécanique tout en maintenant leur densité de matière constante.

Cytosquelette. Réseau dynamique de biopolymères associés à des protéines motrices et des protéines réticulantes. Le cytosquelette impose sa forme à la cellule.

Mécanosension. Réponse et adaptation des cellules aux forces mécaniques locales ou globales ; par exemple, les contacts focaux s'allongent sous une contrainte de cisaillement tout en recrutant de nouvelles protéines, maintenant ainsi leur densité de matière constante.

Moteurs moléculaires. Ces protéines transforment l'énergie chimique donnée par l'hydrolyse de l'Adénosine TriPhosphate en travail mécanique.

Potentiel d'interaction périodique. Les filaments sont périodiques - la même structure se répète tous les $37 \mathrm{~nm}$ pour un filament d'actine - et le potentiel d'interaction périodique entre le moteur et le filament reflète cette périodicité, car l'énergie d'interaction est déterminée par les propriétés locales du filament.

Roue à rochet. Une roue munie d'un cliquet, ou « rochet », ne peut tourner que dans un sens. Dans l'exemple introduit par Richard Feynman pour rectifier un mouvement moléculaire, l'énergie est thermique ; pour la matière vivante considérée dans cet article, la source d'énergie est I'hydrolyse de l'ATP.

Sarcomère et fibre de stress. Le sarcomère est l'unité de base des muscles. II se distingue de la fibre de stress par son haut niveau d'organisation des filaments d'actine et des myosines.

\section{En savoir plus}

1• G. Capello, « Machines moléculaires, allumez vos feux de position! », Reflets de la physique $\mathbf{1 3}$ (mars 2009), 5.

2• F. Julicher, A. Ajdari et J. Prost, "Modelling molecular motors", Rev. Mod. Phys. 69 (1997) 1269.

3• D. Riveline et al., Eur. Biophys. J. 27 (1998) 403.

4• P.Y. Plaçais et al., Phys. Rev. Lett. 103 (2009) 158102.

\footnotetext{
5• K. Kruse et D. Riveline, "Spontaneous mechanical oscillations: implications for developing organisms", Curr. Top. Dev. Biol. 95 (2011) 67.

6• D. Riveline et al., J. Cell Biology 153 (2001) 1175.

7• S.V. Plotnikov et al., Cell 151 (2012) 1513.

8• J. Solon et al., Cell 137 (2009) 1331.
} 